\title{
Recurrence of autoimmune liver diseases after liver transplantation: clinical aspects
}

\author{
Evangelos Cholongitas • Andrew K. Burroughs
}

Received: 25 September 2012/ Accepted: 3 October 2012/Published online: 30 October 2012

(C) Springer-Verlag Italia 2012

\begin{abstract}
Autoimmune hepatitis, primary biliary cirrhosis and primary sclerosing cholangitis are autoimmune liver diseases characterized by progressive immune-mediated inflammation leading to the destruction of the hepatocytes and the biliary epithelial cells, and eventually to cirrhosis and liver failure. The ultimate treatment of these diseases, upon the establishment of end-stage liver disease, includes liver transplantation (LT). Recurrence of autoimmune liver diseases after LT is an entity increasingly recognized in the last few decades. The mechanisms driving recurrence remain poorly understood. The accurate diagnosis of the recurrence and the proper management of the affected patients remains a clinical challenge. This review discusses clinical aspects related to the recurrence of autoimmune liver diseases after LT. The main goals of this review are to discuss the reasons explaining the variability of the incidence rates of recurrent autoimmune disease and the outcome and risk factors for recurrent disease. We discuss in detail the diagnostic criteria and the treatment options of these disorders.
\end{abstract}

Keywords Autoimmune liver disease $\cdot$ Liver transplantation - Recurrence - Autoimmune hepatitis . Primary biliary cirrhosis · Primary sclerosing cholangitis

E. Cholongitas $(\square)$

4th Department of Internal Medicine, Aristotle University

Medical School, Hippokration General Hospital of Thessaloniki,

Thessaloniki, Greece

e-mail: cholongitas@yahoo.gr

A. K. Burroughs

The Sheila Sherlock Liver Centre, University College Institute of

Digestive and Liver Health, Royal Free Hospital, London, UK
Abbreviations
AIH Autoimmune hepatitis
BEC Biliary epithelial cell
LT Liver transplantation
PBC Primary biliary cirrhosis
PSC Primary sclerosing cholangitis
R Recurrent

\section{Introduction}

Recurrence of the primary disease after liver transplantation (LT) has become a major focus for clinicians and researchers. The ultimate goal of the management of these patients is first, to tailor immunosuppression and second, to avoid graft dysfunction and recurrence of the original disease in order to maximize graft survival. Though disease recurrence can be expected to a certain degree for diseases such as viral hepatitides, for others it can be largely unpredictable. This review discusses clinical aspects related to the recurrence of autoimmune liver diseases.

\section{Incidence rates of recurrent autoimmune disease}

Recurrence rates of autoimmune disease after LT are variable in different series, which is partly explained by several differences: (a) methods for the assessment of recurrent disease, (b) criteria used to establish the diagnosis of recurrent disease, (c) use of immunosuppressive regimen and (d) duration of follow-up. It should also be noted that reported rates of recurrence depend on whether routine protocol biopsies are performed, since recurrence disease may be present without abnormal liver function tests. 
Regarding autoimmune hepatitis (AIH), previous studies have reported that recurrent $\mathrm{AIH}$ ( $\mathrm{rAIH}$ ) ranges from 20 to $42 \%$ after LT [1, 2], while a recent review [3] estimated a prevalence rate of $23 \%$ after a median of 26.4 months after LT and a weighted recurrence rate was calculated to be $22 \%$. Recurrence of PSC (rPSC) ranges from 9 to $47 \%$ [4], but in the above-mentioned literature review [3], it was estimated that 161 (17\%) of 940 patients had rPSC, and the weighted recurrence rate was calculated as $11 \%$. Finally, recurrent $\mathrm{PBC}$ (rPBC) has been reported to be approximately $10-35 \%$ at 5 years [5], but its incidence increases with time and in recipients with living donor LT, compared to recipients of cadaveric donor LT [6]. In a recent review [3], an incidence of $16 \%$ was found after a median post-LT follow-up of 69 months and the weighted recurrence rate was $18 \%$ (Table 1 ).

\section{Outcome and risk factors for recurrent disease}

\section{Primary biliary cirrhosis}

The consequences of rPBC appear to be relatively small, since the course of the disease is often, but not always, slow. Generally, rPBC is not considered a major clinical problem [7]. As a result, even in studies with long followup, there was no difference in graft survival between recipients with and those without $\mathrm{rPBC}$. For example, in a series of $485 \mathrm{PBC}$ transplant recipients, recurrent $\mathrm{PBC}$ was the cause of re-LT in only $3(0.6 \%)$ patients [8] and in a

Table 1 Diagnostic criteria for recurrent primary biliary cirrhosis (PBC) after liver transplantation (LT)

\begin{tabular}{l}
\hline Diagnostic criteria for recurrent PBC \\
\hline LT performed for PBC \\
Persistence of AMA or anti-M2 antibody \\
Characteristic portal triad lesions on a liver biopsy ${ }^{\mathrm{a}}$ \\
Epithelioid granulomas \\
Mononuclear inflammatory infiltrate \\
Lymphoid aggregates \\
Bile duct damage \\
Absence of other pathology/disorders, including: \\
Acute and chronic rejection \\
Graft versus host disease \\
Biliary obstruction \\
Vascular abnormalities \\
Cholangitis and other infections \\
Viral hepatitis \\
Drug toxicity
\end{tabular}

a Three of the four portal tract lesions need to be present, and at least three portal fields recent study including 52 patients with $\mathrm{rPBC}$ and extended follow-up after LT to 20 years, it was found that $\mathrm{rPBC}$ had no impact in patient or graft survival. Although patients with $\mathrm{rPBC}$ may have developed more advanced fibrosis, compared to patients transplanted for other liver diseases, it is unclear whether this is clinically relevant. Interestingly, in another cohort, none of 17 patients with $\mathrm{rPBC}$ developed cirrhosis after a mean follow-up of 4.7 years [9]. Risk factors for $\mathrm{rPBC}$ have not elucidated, but advanced donor age, recipients' characteristics and peri-operative factors have been implicated [10]. Regarding immunosupression, the data in the literature remains controversial, but some evidence suggests that cyclosporine-, compared to tacrolimus-based regimen, is associated with reduced rate of $\mathrm{rPBC}$ and slower progression $[8,11,12]$. These reports did not control for the use of azathioprine often combined with cyclosporine and used less often with tacrolimus. In addition, a recent review was not able to confirm that either cyclosporine- or tacrolimus-based immunosuppression were different with respect to long-term survival for PBC patients who had LT [3]. Interestingly, some reports have suggested that steroids may prevent $\mathrm{PBC}$, but their longterm use is associated with higher rates of hypertension, diabetes mellitus and dyslipidemia.

\section{Primary sclerosing cholangitis}

Similarly to PBC, long-term (more than 5 years) patient survival seems to be similar in patients with or without rPSC [13, 14]. However, patients with rPSC underwent more frequently to re-LT due to recurrent disease. Indeed, rPSC is second only to recurrent $\mathrm{HCV}$ in terms of recurrent disease after LT. In addition, PSC graft survival is reduced compared to $\mathrm{PBC}$, which may be related to the slower progression of $\mathrm{PBC}$, compared with PSC. Interestingly, Rowe et al. [15] found that graft loss to be highest in rPSC with a hazard ratio of 6.0 compared to those with $\mathrm{rPBC}$ in a large cohort of patients surviving more than 90 days after LT. Thus, longer follow-up beyond 10 years may be required to assess the impact of rPSC on survival. Recurrence of PSC has been associated with many factors, such as steroid-resistant rejection, OKT3 use, preservation injury, $\mathrm{ABO}$ incompatibility, cytomegalovirus infection, male recipient gender, donor-recipient gender mismatch [16-20] and absence of ulcerative colitis. However, an important issue is the difference in exclusion/inclusion criteria and methods of rPSC diagnosis, which may be the reason for the discrepancies among studies regarding the incidence and the risk factors associated with rPSC [3]. Interestingly, the absence of UC after LT (due to pre-LT colectomy or not) has been found to be an important factor preventing rPSC. In addition, a recent study showed that the presence of severe or de novo UC after LT is a risk 
factor for rPSC [14]. This explains the finding that maintenance steroids ( $>3$ months) (which was the only independent factor for rPSC) was given for activity of UC [14]. These findings give support to the hypotheses that UC and PSC have common pathogenesis [21].

\section{Autoimmune hepatitis}

The course of rAIH has not been elucidated. Although rAIH appears to follow a protracted course with reasonable long-term survival [22], it may be a significant cause of graft loss [15]. These findings have led many centres to suggest long-term immunosuppression with prednisolone at a relatively low dose (about $5-10 \mathrm{mg} /$ day) for at least 1 year post-LT [23]. However, a recent study has suggested that early steroid withdrawal does not influence the incidence of rAIH, and most patients with rAIH can be managed without re-LT [24]. No consistent risk factors for rAIH have been identified, but there are reports that highgrade inflammation in the native liver before LT [25] and HLA-DR3 haplotype are independently associated with higher rate of recurrence [26]. The role of immunosuppression is still controversial, but a recent systematic review by Gautam et al. found no difference in recurrence rates between recipients on tacrolimus or cyclosporinebased regimen $[22,27]$.

\section{Diagnosis of recurrent disease}

\section{Clinical and serological features}

The diagnosis of recurrent autoimmune disease cannot be based on the same criteria as those in the pre-LT setting. For example, it may not reasonable to apply the same scoring system to a LT recipient with suspected recurrent disease, who is under immunosuppression, since many other transplant-related causes may be responsible for the graft dysfunction. On the other hand, the diagnosis of recurrent disease should be based on a combination of biochemical, serological and histological (or radiological) findings. However, abnormal liver tests, detection of autoantibodies and high levels of serum immunoglobulins (IGs) may follow histological changes [28-30]. For example, it is estimated that only half of the patients with rPBC will have abnormal liver tests [9] and the latter may remain normal several years after histological diagnosis of recurrence disease. In addition, auto-antibodies and IGs levels are not useful to establish the diagnosis of recurrence liver disease [2,31]. This is true not only for antimitochondrial antibodies (AMA) in rPBC, but also for other auto-antibodies in patients transplanted for $\mathrm{AIH}$ [30], since the latter have been detected in patients with rejection and in those transplanted for causes other than AIH [30, 32-34].

Primary biliary cirrhosis

Recurrent PBC after LT was first reported in 1982 [35]. Although there was some initial controversy, recurrence of PBC after LT is now well recognized and its diagnosis is based on well-accepted criteria (Table 2) [36]. The diagnosis of $\mathrm{rPBC}$ should be suspected in patients transplanted for PBC and who develop a cholestatic pattern of abnormal hepatic tests or in the presence of typical histopathologic findings during post-LT surveillance. However, histological features of rPBC are often present without abnormal biochemical indices. Thus, a liver biopsy performed only when symptoms or abnormal serum liver tests are present underestimates the rate of rPBC. Thus, many centres perform protocol liver biopsy in order to identify earlier, the patients with rPBC. AMA and anti-M2 are not reliable markers for the presence of $\mathrm{rBBC}$, as they often persist or may only have a transient fall with reappearance after LT without recurrence of PBC. Presumably, they reflect the persistence of the basic abnormality that is associated with development of the disease [28-30, 37]. The gold standard for diagnosing $\mathrm{rPBC}$ is the liver biopsy, demonstrating the characteristic histologic features with granulomatous bile duct destruction with or without plasma cell infiltrate. However, it is important to exclude other processes that can lead to graft dysfunction with similar histologic findings, such as acute or chronic rejection.

Primary sclerosing cholangitis

The first case report suggesting disease recurrence in PSC patients after LT was published in 1988 [38]. The diagnosis

Table 2 Diagnostic criteria for recurrent primary sclerosing cholangitis (PSC) after liver transplantation (LT)

Diagnostic criteria for recurrent PSC

Liver transplantation for PSC

Cholangiography showing non-anastomotic biliary strictures of the intrahepatic and/or extrahepatic biliary system, with irregularities more than 90 days after LT

Liver biopsy specimens showing fibrous cholangitis and/or fibroobliterative lesions with or without ductopaenia

Absence of other pathology/disorders, including:

Recurrent biliary infection

Hepatic artery stenosis or thrombosis

Chronic rejection

Donor/recipient ABO incompatibility

Non-anastomotic stricture developed during the first 90 days after LT 
of rPSC is based on clinical, laboratory, histological, and, mainly, cholangiographic findings, but none of these are specific for rPSC. In addition, a helpful diagnostic serum marker in the pre-LT setting, anti-pANCA is not useful post-LT [39]. The diagnosis is complicated by the fact that other potential causes after LT leading to bile duct lesions suggesting PSC have to be excluded, such as recurrent biliary infection, hepatic artery stenosis or thrombosis, chronic rejection, donor/recipient $\mathrm{ABO}$ incompatibility and ischemic cholangiopathy and/or non-anastomotic strictures developed during the first 90 days after LT, which may mimic PSC in the post-LT setting [39-41]. Day 90 has been chosen in an attempt to eliminate cases of ischemictype biliary stricture related to reperfusion or preservation injury during surgery or quality of the donor graft particularly form non-heart beating donors, which typically develop within that time period [42]. Indeed, rPSC is considered to be a diagnosis of exclusion [13, 43] and strict cholangiographic and/or histological criteria diagnostic of rPSC have been adopted (Table 3) [42]. However, some studies have based the diagnosis of rPSC only on histological findings [44], but it is known that liver biopsy is inaccurate for the diagnosis of PSC due to sampling problems, while other causes, such as ABO incompatibility between donor and recipient and hepatic arterial occlusion, lead to histological lesions that can mimic rPSC. Other studies have been based mainly on radiological demonstration of rPSC with the presence of multiple non-anastomotic strictures seen by magnetic resonance imaging or percutaneous or retrograde cholangiography [45, 46], without considering the fact that ischaemic biliary complications are usually seen up to 6 months post-LT, in contrast with recurrent PSC, which is usually diagnosed more than 12 months after LT. Thus, because of the lack of a diagnostic gold standard, the diagnosis of rPSC after LT remains difficult, and well-defined cholangiographic and histological criteria are mandatory [3, 40, 42].

Table 3 Criteria for the diagnosis of recurrent autoimmune hepatitis (AIH)

Diagnostic criteria for recurrent Autoimmune Hepatitis (AIH)

Liver transplantation for $\mathrm{AIH}$

Serological findings including

Sustained rise in serum aminotransferase activity $(\times 2$ normal $)$

Auto-antibodies in significant titre

Elevated serum immunoglobulins

Diagnostic or compatible liver histology (e.g. plasma cell-rich mononuclear cell portal infiltrate with interface hepatitis)

Absence of other pathology/disorders (e.g. HCV, rejection)
Autoimmune hepatitis

The diagnosis of rAIH is based on increased serum transaminases, and serum $\operatorname{IgG}$ and importantly on appropriate histology showing destruction of liver parenchyma, significant plasma cell infiltration and varying degrees of fibrosis [2]. Alternative diagnoses, such as hepatitis $\mathrm{B}$ and $\mathrm{C}$, and chronic and acute rejection, should be considered before AIH recurrence is diagnosed (Table 4) [22, 47].

\section{Histological features of recurrence}

Histological findings are considered very important, particularly in establishing early the diagnosis of recurrent $\mathrm{AIH}$ and PBC. However, the diagnostic criteria for $\mathrm{AIH}$ and $\mathrm{PBC}$ in the native liver cannot be applied to the liver allograft due to the variable effects of immunosuppression. In addition, there may be histological similarities between recurrent disease and other graft complications, particularly acute and chronic rejection. In $\mathrm{rAIH}$, histological features of a plasma cell-rich mononuclear cell portal infiltrate with interface hepatitis are helpful [48, 49], while the presence of acute lobular hepatitis appears to occur more frequently in rAIH. However, these histological findings should be differentiated from other causes, such as viral infection, de novo $\mathrm{AIH}$ or late cellular rejection with $\mathrm{AIH}$ features [48, 50, 51].

In both PBC and PSC, the classical bile duct lesions are not always seen in liver allograft biopsies. The diagnosis of recurrent $\mathrm{PBC}$ or PSC may be made on the basis of compatible histological findings, such as bile duct loss and features of chronic cholestasis. In PBC, the characteristic portal tract lesions include mononuclear inflammatory infiltrate, formation of lymphoid aggregates, epithelioid granulomas and bile duct damage, but it is important to examine a sufficient number of portal tracts.

Table 4 Characteristics of recurrent autoimmune diseases after liver transplantation

\begin{tabular}{|c|c|c|c|}
\hline & PBC & PSC & $\mathrm{AIH}$ \\
\hline $\begin{array}{l}\text { Recurrence } \\
\text { rate }\end{array}$ & $10-35 \%$ & $9-47 \%$ & $20-42 \%$ \\
\hline Outcome & No effect & Moderate effect & Mild effect \\
\hline Risk factors & $\begin{array}{l}\text { Advanced } \\
\text { donor age, } \\
\text { recipient's } \\
\text { factors } \\
\text { Tacrolimus }\end{array}$ & $\begin{array}{l}\text { Steroid-resistant } \\
\text { rejection, OKT3 } \\
\text { use, } \\
\text { cytomegalovirus } \\
\text { infection, male } \\
\text { recipient gender, } \\
\text { intact colon }\end{array}$ & $\begin{array}{l}\text { High-grade } \\
\text { inflammation } \\
\text { in the native } \\
\text { liver, HLA- } \\
\text { DR3 haplotype }\end{array}$ \\
\hline
\end{tabular}




\section{Treatment}

Although no standard approach exists for the treatment of rPBC, most centres offer patients ursodeoxycholic acid (UDCA) $10-15 \mathrm{mg} / \mathrm{kg} / \mathrm{day}$, as recommended in the pre-LT setting, because of its favourable side-effect profile and efficacy to improve liver biochemistries. In addition, UDCA could delay histologic progression, but its influence on the natural history of recurrent disease requires further study with randomized controlled trials [52]. For example, in one study, 3 (43\%) of 7 patients treated with UDCA for rPBC, had improvement in liver biochemical tests, while in another study, $13(75 \%)$ of 17 patients with rPBC treated with UDCA showed a marked decrease in serum alkaline phosphatase. However, it is unknown whether the biochemical improvement correlated with a histologic improvement [5], and there have been no prospective, controlled trial. Indeed, the precise benefit of UDCA in this setting remains unknown. On the other hand, since the incidence of colon cancer increases after LT, similarly to PSC, UDCA may reduce the risk of dysplasia and thus colon cancer. The benefit of using UDCA in combination with corticosteroids (such as budesonide, which has an $90 \%$ first-pass metabolism by the liver) has not elucidated in the post-LT setting [53]. Although optimal immunosuppression to prevent $\mathrm{PBC}$ recurrence remains controversial, it has been reported that recurrence of PBC in patients receiving tacrolimus is more rapid than with cyclosporine, but the use of azathioprine was not controlled in this analysis. Switching to alternative regimes (e.g. those based on azathioprine, mycophenolate or cyclosporine) may be an option if recurrence appears to progress rapidly.

In the pre-LT setting, no specific treatment has been shown to prevent or slow progression of PSC. High dose of UDCA (15-20 mg/kg/day) may be detrimental [54]. Particularly, UDCA might benefit more those patients with PSC and UC by reducing the risk of dysplasia leading to colon adenoma and carcinoma. Based on these data and the safety of UDCA, UDCA is often used in rPSC, while the choice of immunosuppression does not seem to have any influence on recurrence.

In $\mathrm{rAIH}$, the general approach is similar to that of $\mathrm{AIH}$ pre-LT, i.e. to increase corticosteroids up to $20 \mathrm{mg} /$ day (with or without azathioprine) and in non-responders switching from a cyclosporine to tacrolimus-based regimen[55]. The addition of mycophenolate $2 \mathrm{~g}$ /day had been effective in some non-responders. The calcineurin inhibitors (CNIs) and mycophenolate have been used to treat $\mathrm{AIH}$ in the native liver, and may be effective in the graft. Treatment should be guided by the liver tests, levels of $\mathrm{IgG}$, auto-antibodies and liver histology. However, not all patients respond to enhanced immunosuppression. Eventually, re-LT may be required in some patients.
Transplantation centres commonly maintain AIH patients on prednisone and/or higher dose immunosuppression than non-AIH patients after LT in order to reduce rejection and recurrence rates, but the usefulness of this approach requires further evaluation.

Conflict of interest None.

\section{References}

1. Gonzalez-Koch A, Czaja AJ, Carpenter HA et al (2001) Recurrent autoimmune hepatitis after orthotopic liver transplantation. Liver Transpl 7:302-310

2. Vogel A, Heinrich E, Bahr MJ et al (2004) Long-term outcome of liver transplantation for autoimmune hepatitis. Clin Transplant 18:62-69

3. Gautam M, Cheruvattath R, Balan V (2006) Recurrence of autoimmune liver disease after liver transplantation: a systematic review. Liver Transpl 12:1813-1824

4. Kotlyar DS, Campbell MS, Reddy KR (2006) Recurrence of diseases following orthotopic liver transplantation. Am J Gastroenterol 101:1370-1378

5. Guy JE, Qian P, Lowell JA et al (2005) Recurrent primary biliary cirrhosis: peritransplant factors and ursodeoxycholic acid treatment post-liver transplant. Liver Transpl 11:1252-1257

6. Yamagiwa S, Ichida T (2007) Recurrence of primary biliary cirrhosis and primary sclerosing cholangitis after liver transplantation in Japan. Hepatol Res 37(Suppl 3):S449-S454

7. Jacob DA, Neumann UP, Bahra M et al (2006) Long-term followup after recurrence of primary biliary cirrhosis after liver transplantation in 100 patients. Clin Transplant 20:211-220

8. Neuberger J, Gunson B, Hubscher S et al (2004) Immunosuppression affects the rate of recurrent primary biliary cirrhosis after liver transplantation. Liver Transpl 10:488-491

9. Sylvestre PB, Batts KP, Burgart LJ et al (2003) Recurrence of primary biliary cirrhosis after liver transplantation: histologic estimate of incidence and natural history. Liver Transpl 9:1086-1093

10. Liermann Garcia RF, Evangelista GC, McMaster P et al (2001) Transplantation for primary biliary cirrhosis: retrospective analysis of 400 patients in a single center. Hepatology 33:22-27

11. Levitsky J, Hart J, Cohen SM et al (2003) The effect of immunosuppressive regimens on the recurrence of primary biliary cirrhosis after liver transplantation. Liver Transpl 9:733-736

12. Sanchez EQ, Levy MF, Goldstein RM et al (2003) The changing clinical presentation of recurrent primary biliary cirrhosis after liver transplantation. Transplantation 76:1583-1588

13. Graziadei IW (2002) Recurrence of primary sclerosing cholangitis after liver transplantation. Liver Transpl 8:575-581

14. Cholongitas E, Shusang V, Papatheodoridis GV et al (2008) Risk factors for recurrence of primary sclerosing cholangitis after liver transplantation. Liver Transpl 14:138-143

15. Rowe IA, Webb K, Gunson BK et al (2008) The impact of disease recurrence on graft survival following liver transplantation: a single centre experience. Transpl Int 21:459-465

16. Brandsaeter B, Schrumpf E, Bentdal O et al (2005) Recurrent primary sclerosing cholangitis after liver transplantation: a magnetic resonance cholangiography study with analyses of predictive factors. Liver Transpl 11:1361-1369

17. Khettry U, Keaveny A, Goldar-Najafi A et al (2003) Liver transplantation for primary sclerosing cholangitis: a long-term clinicopathologic study. Hum Pathol 34:1127-1136 
18. Kugelmas M, Spiegelman P, Osgood MJ et al (2003) Different immunosuppressive regimens and recurrence of primary sclerosing cholangitis after liver transplantation. Liver Transpl 9:727-732

19. Vera A, Moledina S, Gunson B et al (2002) Risk factors for recurrence of primary sclerosing cholangitis of liver allograft. Lancet 360:1943-1944

20. Jeyarajah DR, Netto GJ, Lee SP et al (1998) Recurrent primary sclerosing cholangitis after orthotopic liver transplantation: is chronic rejection part of the disease process? Transplantation 66:1300-1306

21. Cholongitas E, Shusang V, Patch D et al (2008) Pathogenesis of primary sclerosing cholangitis. J Hepatol 49:863-864

22. Molmenti EP, Netto GJ, Murray NG et al (2002) Incidence and recurrence of autoimmune/alloimmune hepatitis in liver transplant recipients. Liver Transpl 8:519-526

23. Neuberger J, Jothimani D (2005) Long-term immunosuppression for prevention of nonviral disease recurrence. Transpl Proc 37:1671-1674

24. Campsen J, Zimmerman MA, Trotter JF et al (2008) Liver transplantation for autoimmune hepatitis and the success of aggressive corticosteroid withdrawal. Liver Transpl 14:1281-1286

25. Ayata G, Gordon FD, Lewis WD et al (2000) Liver transplantation for autoimmune hepatitis: a long-term pathologic study. Hepatology 32:185-192

26. Wright HL, Bou-Abboud CF, Hassanein T et al (1992) Disease recurrence and rejection following liver transplantation for autoimmune chronic active liver disease. Transplantation 53:136-139

27. Heffron TG, Smallwood GA, Oakley B et al (2002) Autoimmune hepatitis following liver transplantation: relationship to recurrent disease and steroid weaning. Transplant Proc 34:3311-3312

28. Ahmed M, Mutimer D, Hathaway M et al (1997) Liver transplantation for autoimmune hepatitis: a 12-year experience. Transplant Proc 29:496

29. Gotz G, Neuhaus R, Bechstein WO et al (1999) Recurrence of autoimmune hepatitis after liver transplantation. Transpl Proc $31: 430-431$

30. Duclos-Vallee JC, Sebagh M, Rifai K et al (2003) A 10 year follow up study of patients transplanted for autoimmune hepatitis: histological recurrence precedes clinical and biochemical recurrence. Gut 52:893-897

31. Haydon GH, Neuberger J (2000) PBC: an infectious disease? Gut 47:586-588

32. Lohse AW, Obermayer-Straub P, Gerken G et al (1999) Development of cytochrome P450 2D6-specific LKM-autoantibodies following liver transplantation for Wilson's disease-possible association with a steroid-resistant transplant rejection episode. J Hepatol 31:149-155

33. Jones DE, James OF, Portmann B et al (1999) Development of autoimmune hepatitis following liver transplantation for primary biliary cirrhosis. Hepatology 30:53-57

34. Kerkar N, Hadzic N, Davies ET et al (1998) De novo autoimmune hepatitis after liver transplantation. Lancet 351:409-413

35. Neuberger J, Portmann B, Macdougall BR et al (1982) Recurrence of primary biliary cirrhosis after liver transplantation. N Engl J Med 306:1-4

36. Hubscher SG, Elias E, Buckels JA et al (1993) Primary biliary cirrhosis. Histological evidence of disease recurrence after liver transplantation. J Hepatol 18:173-184

37. Gouw AS, Haagsma EB, Manns M et al (1994) Is there recurrence of primary biliary cirrhosis after liver transplantation? A clinicopathologic study in long-term survivors. J Hepatol 20:500-507
38. Lerut J, Demetris AJ, Stieber AC et al (1988) Intrahepatic bile duct strictures after human orthotopic liver transplantation. Recurrence of primary sclerosing cholangitis or unusual presentation of allograft rejection? Transpl Int 1:127-130

39. Faust TW (2001) Recurrent primary biliary cirrhosis, primary sclerosing cholangitis, and autoimmune hepatitis after transplantation. Liver Transpl 7:S99-S108

40. LaRusso NF, Shneider BL, Black D et al (2006) Primary sclerosing cholangitis: summary of a workshop. Hepatology 44:746-764

41. Gordon F (2006) Recurrent primary sclerosing cholangitis: clinical diagnosis and long-term management issues. Liver Transpl 12(Suppl 2):S73-S75

42. Graziadei IW, Wiesner RH, Batts KP et al (1999) Recurrence of primary sclerosing cholangitis following liver transplantation. Hepatology 29:1050-1056

43. Graziadei IW, Wiesner RH, Marotta PJ et al (1999) Long-term results of patients undergoing liver transplantation for primary sclerosing cholangitis. Hepatology 30:1121-1127

44. Harrison RF, Davies MH, Neuberger JM et al (1994) Fibrous and obliterative cholangitis in liver allografts: evidence of recurrent primary sclerosing cholangitis? Hepatology 20:356-361

45. Sheng R, Zajko AB, Campbell WL et al (1993) Biliary strictures in hepatic transplants: prevalence and types in patients with primary sclerosing cholangitis vs those with other liver diseases. AJR Am J Roentgenol 161:297-300

46. Sheng R, Campbell WL, Zajko AB et al (1996) Cholangiographic features of biliary strictures after liver transplantation for primary sclerosing cholangitis: evidence of recurrent disease. AJR Am J Roentgenol 166:1109-1113

47. Manns MP, Bahr MJ (2000) Recurrent autoimmune hepatitis after liver transplantation-when non-self becomes self. Hepatology $32: 868-870$

48. Hubscher SG (2001) Recurrent autoimmune hepatitis after liver transplantation: diagnostic criteria, risk factors, and outcome. Liver Transpl 7:285-291

49. Milkiewicz P, Gunson B, Saksena S et al (2000) Increased incidence of chronic rejection in adult patients transplanted for autoimmune hepatitis: assessment of risk factors. Transplantation 70:477-480

50. Krasinskas AM, Demetris AJ, Poterucha JJ et al (2008) The prevalence and natural history of untreated isolated central perivenulitis in adult allograft livers. Liver Transpl 14:625-632

51. Sundaram SS, Melin-Aldana H, Neighbors K et al (2006) Histologic characteristics of late cellular rejection, significance of centrilobular injury, and long-term outcome in pediatric liver transplant recipients. Liver Transpl 12:58-64

52. Charatcharoenwitthaya P, Pimentel S, Talwalkar JA et al (2007) Long-term survival and impact of ursodeoxycholic acid treatment for recurrent primary biliary cirrhosis after liver transplantation. Liver Transpl 13:1236-1245

53. Rautiainen H, Karkkainen P, Karvonen AL et al (2005) Budesonide combined with UDCA to improve liver histology in primary biliary cirrhosis: a three-year randomized trial. Hepatology 41:747-752

54. Pardi DS, Loftus EV Jr, Kremers WK et al (2003) Ursodeoxycholic acid as a chemopreventive agent in patients with ulcerative colitis and primary sclerosing cholangitis. Gastroenterology 124:889-893

55. Hurtova M, Duclos-Vallee JC, Johanet C et al (2001) Successful tacrolimus therapy for a severe recurrence of type 1 autoimmune hepatitis in a liver graft recipient. Liver Transpl 7:556-558 